\title{
Perfil nutricional de portadores de HIV/AIDS residentes no Brasil
}

\author{
Nutritional profile of HIV/AIDS patients residing in Brazil
}

\section{Perfil nutricional de los pacientes con VIH/SIDA residentes en Brasil}

Fernanda Keila Valente Batista ${ }^{1 *}$, Scarlett Valente Batista ${ }^{1}$, Ana Ranyele de Oliveira Pereira ${ }^{1}$, Larissa Costa e Silva ${ }^{1}$, Perla Silva Rodrigues ${ }^{1}$, Leno Rafael Lima Freire ${ }^{1}$, Ívina Michelle Silva Peixoto ${ }^{1}$, Antônia Anna Lídia Ferreira Ribeiro ${ }^{1}$, Débora Bezerra Xavier ${ }^{1}$.

\section{RESUMO}

Objetivo: Realizar uma revisão de literatura a fim de reunir as evidências científicas acerca do perfil nutricional de portadores de HIV/AIDS residentes no Brasil. Métodos: Revisão integrativa, realizada por meio de busca nas bases de dados: Literatura Latino-Americana e do Caribe em Ciências da Saúde (LILACS), na Biblioteca Virtual em Saúde (BVS), PubMed e Acervo Mais. Os descritores utilizados para a pesquisa foram selecionados mediante consulta ao DECS/Mesh (Descritores em Ciências da Saúde), sendo eles "estado nutricional", "HIV" e "AIDS", além de seus termos correspondentes na língua inglesa. Resultados: Fizeram parte da amostra final 16 artigos. Os achados revelaram que embora nos últimos anos tenha ocorrido uma mudança no perfil nutricional desse público, com o aumento de indivíduos eutróficos, a desnutrição permanece com uma prevalência elevada, notou-se também um aumento do excesso de peso nessa população. Considerações finais: Os resultados dos estudos mostram que, embora exista uma mudança no padrão corporal desse público, com o aumento de eutrofia e excesso de peso, a desnutrição permanece muito presente nos portadores de HIV/AIDS.

Palavras-chave: Estado nutricional, HIV, AIDS.

\section{ABSTRACT}

Objective: Perform a literature review in order to gather scientific evidence about the nutritional profile of HIV / AIDS patients residing in Brazil. Methods: Systematic review, carried out by searching the databases: Latin American and Caribbean Literature in Health Sciences (LILACS), the Virtual Health Library (VHL), PubMed and Acervo Mais. The descriptors used for the research were selected by consulting the DECS / Mesh (Health Sciences Descriptors), which are "nutritional status", "HIV" and "AIDS", in addition to their corresponding terms in English. Results: 16 articles were part of the final sample. The findings revealed that although there has been a change in the nutritional profile of this public in recent years, with the increase in eutrophic individuals, malnutrition remains with a high prevalence, there has also been an increase in overweight in this population. Final considerations: The results of the studies show that, although there is a change in the body pattern of this public, with the increase in eutrophy and overweight, malnutrition remains very present in people with HIV / AIDS.

Keywords: Nutritional status, HIV, AIDS.

\section{RESUMEN}

Objetivo: Realizar una revisión de la literatura para recopilar evidencia científica sobre el perfil nutricional de los pacientes con VIH / SIDA que residen en Brasil. Métodos: Revisión sistemática, realizada mediante la búsqueda en las bases de datos: Literatura Latinoamericana y del Caribe en Ciencias de la Salud (LILACS), Biblioteca Virtual en Salud (BVS), PubMed y Acervo Mais. Los descriptores utilizados para la investigación fueron seleccionados consultando los DECS / Mesh (Descriptores de Ciencias de la Salud), que son "estado nutricional", "VIH" y "SIDA", además de sus términos correspondientes en inglés. Resultados: 16 artículos formaron parte de la muestra final. Los hallazgos revelaron que si bien se ha producido un cambio en el perfil nutricional de este público en los últimos años, con el aumento de los individuos eutróficos, la desnutrición se mantiene con una alta prevalencia, también se ha producido un aumento del sobrepeso en esta población. Consideraciones finales: Los resultados de los estudios muestran que, aunque hay un cambio en el patrón corporal de este público, con el aumento de la eutrofia y el sobrepeso, la desnutrición sigue muy presente en las personas con VIH / SIDA.

Palabra clave: Estado nutricional, VIH, SIDA.

1 Instituto Federal de Educação, Ciência e Tecnologia do Ceará (IFCE), Limoeiro do Norte - CE.

*E-mail: fernandakvb@hotmail.com

SUBMETIDO EM: 12/2020 


\section{INTRODUÇÃO}

O Vírus da Imunodeficiência Humana (HIV) é um vírus que provoca danos no sistema imunológico de seus portadores, causando infecção de células importantes do organismo, ocasionando, portanto, uma grave supressão de células de defesa. É considerado um retrovírus que converte o RNA em DNA, desse modo, faz com que as células do organismo o reconheçam e ele se incorpore ao cromossomo das mesmas. Existem diversas formas pela a qual o vírus do HIV é capaz de penetrar no organismo humano, sendo elas: por meio de relação sexual sem preservativo, sendo essa uma das principais formas de transmissão do vírus, transmissão vertical, aleitamento materno, agulhas injetáveis e objetos pérfuro cortantes. Esse distúrbio caracteriza a Síndrome da Imunodeficiência Adquirida (AIDS), que evolui devido a uma significativa destruição de linfócitos TCD4+ (PEREIRA MD, et al., 2019; SANTANA JC, et al., 2019).

A síndrome da imunodeficiência adquirida (AIDS) é uma patologia que se expressa no organismo após a infecção pelo vírus HIV, ou seja, o HIV precede a AIDS e nunca o contrário. A AIDS é uma doença letal, sendo transmitida do sangue contaminado, é considerada uma patologia crônica, que consiste em um grave problema de saúde pública, pois leva o indivíduo a alterações importantes no estado nutricional e imunológico. O estado nutricional debilitado dos portadores de AIDS provoca a supressão da função imunocelular, que leva ao aparecimento das infecções oportunistas, sendo estas, as causas primárias de mortes em pacientes com HIV/AIDS. Entretanto, já é de conhecimento que a nutrição possibilita a obtenção de melhores resultados no combate à AIDS (PINTO AF, et al., 2016).

Segundo o último Boletim Epidemiológico de HIV e AIDS, divulgado pelo Ministério da Saúde, no Brasil, no ano de 2018, foram diagnosticados 43.941 novos casos de HIV e 37.161 casos de AIDS, notificados no Sinan (Sistema de Informação de Agravos de Notificação), com uma taxa de detecção de 17,8/100.000 habitantes, totalizando assim, no período de 1980 a junho de 2019, 966.058 casos de AIDS detectados em todo país, revelando, que muitas pessoas convivem com o vírus (BRASIL, 2019).

A doença evoluí a partir de distúrbios que causam infecções oportunistas, alterações nutricionais e até a morte do indivíduo portador do vírus. O HIV é uma patologia sexualmente transmissível que acomete milhares de pessoas no Brasil e no mundo, independente de raça, gênero e condição econômica. $O$ tratamento com antirretroviral (TARV) prolonga a vida das pessoas que vivem com HIV/AIDS, visto que, os medicamentos impedem que ocorra a replicação viral do HIV, fazendo assim, com que haja um aumento no número de linfócitos CD4+, que são células de defesa do organismo humano capazes de destruir os antígenos causadores das diversas doenças oportunistas, reduzindo o risco das infecções por essas doenças, associado a isso, reduz a chance de transmissão, além do TARV colaborar para o aumento de expectativa de vida desse público (PEREIRA MD, et al., 2019; COSTA CS, et al., 2017; SANTANA JC, et al., 2019).

O trato gastrointestinal é considerado o primeiro e mais prejudicado órgão em decorrência da síndrome, sobretudo, na parte que integra o tecido imunológico. Essas alterações provocam agravos que afetam desde a mastigação até a absorção de nutrientes. Geralmente os pacientes apresentam apetite diminuído e ingestão energética insuficiente, associada a um gasto energético de repouso aumentado, a soma desses fatores provocam uma importante perda de tecido adiposo e massa muscular. Devido a essas alterações fisiológicas, realizar avaliação nutricional nos portadores do vírus é indispensável para rastrear precocemente o risco nutricional e intervir eficazmente (PEREIRA MD, et al., 2019).

Não obstante, a avaliação do estado nutricional desses pacientes deve ser constante, afim de se manter os cuidados nutricionais, evitando uma piora do quadro e a suscetibilidade a complicações, sabendo, que uma alimentação saudável e adequada em nutrientes contribui com a melhora da qualidade de vida, além de permitir ao profissional de saúde, compreender melhor algumas condições que ajudam no enfrentamento da patologia a partir de uma intervenção nutricional adequada, visando a manutenção ou recuperação do estado nutricional (PINTO AF, et al., 2016).

Existem diferentes métodos que são utilizados para avaliar o estado nutricional de um indivíduo, esses métodos podem ser objetivos ou subjetivos. Os objetivos compreendem, a antropometria, composição corporal, exames bioquímicos e consumo alimentar, enquanto os subjetivos, englobam o exame físico e a 
triagem nutricional. As medidas antropométricas mais utilizadas são o peso e a estatura, que podem ser aferidos ou estimados, as circunferências e as dobras cutâneas. A triagem nutricional consiste em uma avaliação sistemática com o intuito de investigar a existência de características que podem estar relacionadas, ou que refletem, a deterioração nutricional (KAMIMURA MA, et al., 2014).

Assim, o estudo justifica-se pela necessidade possuir conhecimento sobre o perfil nutricional desse público, a fim de rastrear e identificar o nível de vulnerabilidade nutricional em que essa população está inserida, possibilitando dessa forma que profissionais responsáveis pelo cuidado nutricional acompanhem rigorosamente esses pacientes, evitando complicações, aumento da morbimortalidade, e possibilite, inclusive, a busca por estratégias para prevenir ou recuperar o estado nutricional de portadores do vírus HIV, uma vez que a desnutrição nesse público aumenta o risco de evolução para a fase mais avançada da infecção causada pelo vírus HIV, a AIDS.

Dito isto, o objetivo desse estudo é realizar uma revisão integrativa com a finalidade de reunir as evidências científicas acerca do perfil nutricional de portadores de HIV/AIDS, residentes no Brasil.

\section{MÉTODOS}

O presente estudo trata-se de uma revisão integrativa de literatura, realizada por meio de busca nas bases de dados eletrônicos: LILACS, BVS, PubMed e Acervo Mais. Os descritores utilizados para a pesquisa foram selecionados mediante consulta ao DECS/Mesh (Descritores em Ciências da Saúde), sendo eles "estado nutricional", "HIV" e "AIDS", além de seus termos correspondentes na língua inglesa. As pesquisas foram realizadas com os termos combinados entre si.

Utilizou-se como critérios de inclusão artigos científicos publicados nos idiomas português e inglês, publicados entre os anos de 2010 a 2020 e que se relacionassem ao objetivo deste artigo, ou seja, artigos que avaliassem e fornecessem dados sobre o perfil nutricional de portadores de HIV/AIDS. Em relação aos critérios de exclusão, foram excluídos trabalhos disponíveis em outros idiomas que não os elegidos, trabalhos que não estavam disponíveis na íntegra, referências duplicadas e/ou que não atendessem aos critérios de inclusão pré-estabelecidos.

Após realizar as buscas nas bases de dados utilizando os descritores e aplicando os critérios de inclusão, obteve-se 648 artigos. Após a leitura dos títulos e resumos, 44 foram selecionados e lidos na íntegra, destes, 16 compuseram a amostra final da revisão. Pode-se observar em síntese o processo de busca e seleção dos artigos (Figura 1).

Figura 1 - Processo de seleção dos artigos.

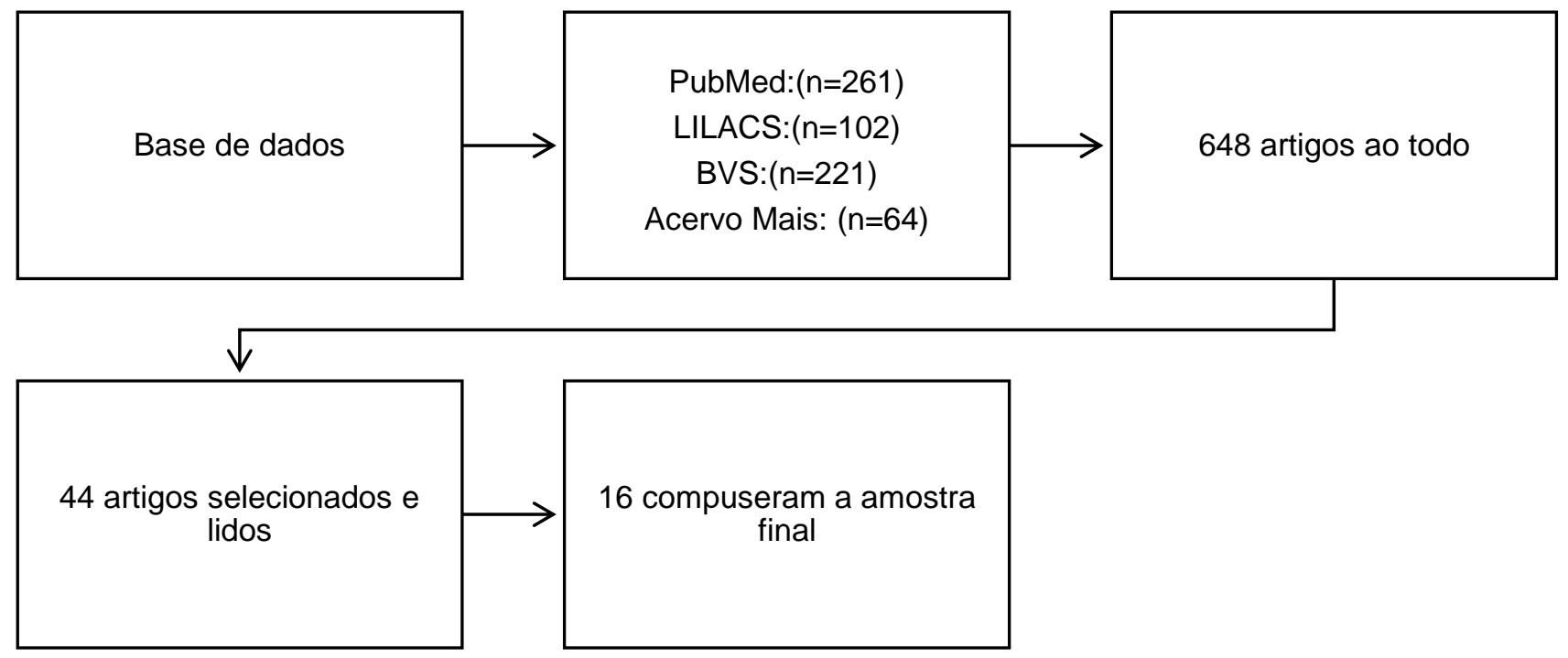

Fonte: Batista FKV, et al., 2020. 


\section{RESULTADOS}

O tipo de estudo prevalente foi o transversal, presente em 12 artigos, e a população-alvo mais frequente foram os adultos. A maior parte dos estudos utilizou mais de um método para realizar o diagnóstico nutricional $(n=12)$. Os manuscritos analisados realizaram o diagnostico nutricional através de métodos objetivos e subjetivos. O método subjetivo que os autores utilizaram para realizar o diagnóstico foram as ferramentas de triagem nutricional, utilizadas em 2 estudos. $O$ método objetivo utilizado pelos autores na realização das pesquisas foi a antropometria, com a realização da aferição do peso e estatura, que foram utilizados para realizar o cálculo do índice de massa corporal (IMC) e com a mensuração da circunferência do braço (CB) e da dobra cutânea tricipital (DCT).

Como já citado, dos 16 artigos selecionados para compor a amostra, 12 utilizaram mais de um método para realizar o diagnóstico nutricional dos participantes, os 4 restantes optaram por utilizar apenas um método. Dos 12 estudos que utilizaram mais de um método 8 não concordaram no diagnostico prevalente, nesses estudos o IMC diagnosticou que a população estava eutrófica, enquanto a CB, DCT e ANSG, que foram os outros métodos utilizados nos estudos, diagnosticaram que a maior parte da população se encontrava com algum grau de desnutrição.

Nos estudos restantes, que também realizaram o diagnostico nutricional através de mais de um método, concordaram com o diagnostico nutricional prevalente, que no caso foi a desnutrição. Já os estudos que utilizaram apenas um método, nesses artigos o estado nutricional que prevaleceu foi a eutrofia. De uma forma geral, os estados nutricionais prevalentes nas populações estudadas foram, a eutrofia e a desnutrição, a depender da medida utilizada, sendo o IMC à medida que mais diagnosticou estado nutricional adequado, e a CB juntamente com a DCT foram as medidas que mais diagnosticaram desnutrição.

A seguir encontra-se disposto a caracterização do conteúdo dos artigos analisados segundo autores, ano de publicação, local de realização do estudo, delineamento, faixa etária da população estudada e principais resultados acerca do estado nutricional dos portadores de HIV/AIDS (Quadro 1). 


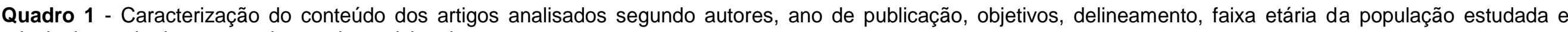
principais resultados acerca do estado nutricional.

\begin{tabular}{|c|c|c|c|c|c|}
\hline $\begin{array}{l}\text { Autor/Ano de } \\
\text { publicação }\end{array}$ & $\begin{array}{l}\text { Cidade, Unidade } \\
\text { Federativa }\end{array}$ & Delineamento & Faixa etária & $\begin{array}{c}\text { Método } \\
\text { utilizado para } \\
\text { avaliar o } \\
\text { estado EN }\end{array}$ & $\begin{array}{l}\text { Diagnóstico nutricional prevalente em cada } \\
\text { método }\end{array}$ \\
\hline Pinto AF, et al., (2016) & Belém, Pará & Transversal analítico & 20 a 59 anos & $\begin{array}{l}-\mathrm{IMC} \\
-\mathrm{CB} \\
-\mathrm{DCT}\end{array}$ & $\begin{array}{l}\text {-IMC: Desnutrição }(54,3 \%) \\
\text {-CB: Desnutrição }(78,3 \%) \\
\text {-DCT: Desnutrição }(91,3 \%)\end{array}$ \\
\hline $\begin{array}{l}\text { Silva VS, Mori RMSC e } \\
\text { Guimarães SM (2012) }\end{array}$ & Belém, Pará & $\begin{array}{l}\text { Descritivo de corte } \\
\text { transversal }\end{array}$ & 20 a 59 anos & $\begin{array}{l}-I M C \\
-C B \\
-D C T\end{array}$ & $\begin{array}{l}\text {-IMC: Desnutrição }(53,2 \%) \\
\text {-CB: Desnutrição }(65,5 \%) \\
\text {-DCT: Desnutrição }(46,3 \%)\end{array}$ \\
\hline $\begin{array}{l}\text { Ladeira POC e Silva } \\
\text { DCG (2012) }\end{array}$ & Itaperuna, Rio de Janeiro & Transversal & 20 a 59 anos & $\begin{array}{l}-I M C \\
-D C T\end{array}$ & $\begin{array}{c}\text {-IMC: Eutrofia (54\%) } \\
\text { Excesso de peso }(38 \%) \\
\text {-DCT: desnutrição grave }(51,5 \%)\end{array}$ \\
\hline Sllva AAA, et al., (2015) & Porto Velho, Rondônia & Transversal & 23 a 58 anos & $\begin{array}{l}-\mathrm{IMC} \\
-\mathrm{CB}\end{array}$ & $\begin{array}{c}\text {-IMC: Eutrofia: (65\%) } \\
\text {-CB: Algum grau de desnutrição: (80\%) }\end{array}$ \\
\hline Souza CN et al., (2017) & $\begin{array}{l}\text { Campo Grande, Mato } \\
\text { Grosso do Sul }\end{array}$ & Descritivo quantitativo & 18 a 59 anos & $\begin{array}{l}-\mathrm{IMC} \\
-\mathrm{CB}\end{array}$ & $\begin{array}{l}\text {-IMC: Desnutrição: (55\%) } \\
\text {-CB: Algum grau de desnutrição: (85\%) }\end{array}$ \\
\hline $\begin{array}{l}\text { Kauffmann LKO, al., } \\
\qquad(2016)\end{array}$ & Belém, Pará & Transversal, descritivo & 20 a 59 anos & $\begin{array}{l}-I M C \\
-C B \\
-D C T\end{array}$ & $\begin{array}{l}\text {-IMC: Desnutrição }(55,5 \%) \\
\text {-CB: Desnutrição }(79,6 \%) \\
\text {-DCT: Desnutrição }(92,6 \%)\end{array}$ \\
\hline $\begin{array}{l}\text { Oliveira } R L \text {, et al., } \\
(2018)\end{array}$ & $\begin{array}{l}\text { Rio de Janeiro, Rio de } \\
\text { Janeiro }\end{array}$ & Estudo seccional & 18 a 60 anos & -ASNG & $\begin{array}{c}\text { ANSG: Bem nutrido }(69,9 \%) \\
\text { Desnutrição leve ou moderada }(30,1 \%)\end{array}$ \\
\hline $\begin{array}{c}\text { Rodrigues EC, Miranda } \\
\text { RNA e Guterres AS } \\
\text { (2013) }\end{array}$ & Belém, Pará & Transversal & 18 a 59 anos & $\begin{array}{l}-I M C \\
-C B \\
-D C T\end{array}$ & $\begin{array}{l}\text {-IMC: Eutrofia }(53,4 \%) \\
\text { Excesso de peso }(39,3 \%) \\
\text {-CB: desnutrição }(46,7 \%) \\
\text {-DCT: Desnutrição }(74 \%)\end{array}$ \\
\hline Pires DS, et al., (2017) & Goiânia, Goiás & Transversal & 20 a 61 anos & $\begin{array}{l}- \text { IMC } \\
-\mathrm{CB} \\
\text {-DCT } \\
\text {-ANSG }\end{array}$ & $\begin{array}{c}\text {-IMC: Eutrofia (60\%) } \\
\text {-CB: Desnutrição }(75,7 \%) \\
\text {-DCT: Desnutrição }(74,3 \%) \\
\text {-ANSG: Algum grau de desnutrição (52\%) }\end{array}$ \\
\hline
\end{tabular}




\begin{tabular}{|c|c|c|c|c|c|}
\hline $\begin{array}{l}\text { Autor/Ano de } \\
\text { publicação }\end{array}$ & $\begin{array}{l}\text { Cidade, Unidade } \\
\text { Federativa }\end{array}$ & Delineamento & Faixa etária & $\begin{array}{l}\text { Método } \\
\text { utilizado para } \\
\text { avaliar o } \\
\text { estado EN }\end{array}$ & $\begin{array}{l}\text { Diagnóstico nutricional prevalente em cada } \\
\text { método }\end{array}$ \\
\hline $\begin{array}{l}\text { Bassichetto KC, et al., } \\
\text { (2014) }\end{array}$ & São Paulo, São Paulo & Corte transversal & 20 a 74 anos & $-I M C$ & $\begin{array}{c}\text { Eutrofia: } 54,1 \% \\
\text { Excesso de peso: } 38,6 \%\end{array}$ \\
\hline Kroll AF, et al., (2012) & $\begin{array}{l}\text { Porto Alegre, Rio Grande } \\
\text { do Sul }\end{array}$ & Transversal & $>18$ anos & $-I M C$ & $\begin{array}{c}\text { Eutrofia: } 53,2 \% \\
\text { Excesso de peso: } 42,6 \%\end{array}$ \\
\hline $\begin{array}{l}\text { Braga LA e Silva CAB } \\
\qquad(2010)\end{array}$ & Fortaleza, Ceará & Transversal e descritivo & 19 a 65 anos & $\begin{array}{l}-\mathrm{IMC} \\
-\mathrm{CB} \\
-\mathrm{DCT}\end{array}$ & $\begin{array}{c}\text {-IMC: Eutrofia }(60 \%) \\
\text { Sobrepeso }(22,8 \%) \\
\text {-CB: Algum grau de desnutrição }(53 \%) \\
\text {-DCT: Algum grau de desnutrição }(72,8 \%)\end{array}$ \\
\hline Costa CS, et al., (2017) & Fortaleza, Ceará & $\begin{array}{c}\text { Transversal, descritivo e } \\
\text { quantitativo }\end{array}$ & 23 a 55 anos & $\begin{array}{c}-I M C \\
- \text { CB } \\
- \text { ANSG } \\
\text {-NRS-2002 } \\
\text {-MUST }\end{array}$ & $\begin{array}{c}\text {-IMC: Eutrofia }(43,3 \%) \\
\text { Algum grau de desnutrição }(40,1 \%) \\
\text {-CB: Algum grau de desnutrição }(89,3 \%) \\
\text {-ANSG: Bem nutrido (86\%) } \\
\text { - NRS-2002: Risco nutricional }(56,7 \%) \\
\text {-MUST: Alto risco }(66,3 \%)\end{array}$ \\
\hline Oliveira N, et al, (2019) & Ouro Preto, Minas Gerais & Transversal & $\geq 18$ & $\begin{array}{l}-I M C \\
-C D \\
-D C T\end{array}$ & $\begin{array}{c}\text {-IMC: Eutrofia }(48,6 \%) \\
\text { Excesso de peso }(37,1 \%) \\
\text {-CB: Algum grau de desnutrição }(48,6 \%) \\
\text {-DCT: Algum grau de desnutrição }(57,1 \%) \text { e } \\
37,4 \% \text { com excesso de peso }\end{array}$ \\
\hline Aires IO, et al., (2019) & Teresina, Piauí & Relatos de casos & $\geq 20$ anos & $-I M C$ & $\begin{array}{l}\text { Dos } 8 \text { participantes do estudo, } 4 \text { estavam } \\
\text { eutróficas; } 3 \text { estavam com sobrepeso ou } \\
\text { obesidade e apenas } 1 \text { foi diagnosticado com } \\
\text { baixo peso. }\end{array}$ \\
\hline $\begin{array}{l}\text { Miranda RNA, et al., } \\
\qquad(2019)\end{array}$ & Belém, Pará & transversal, prospectivo & 19 a 34 anos & $\begin{array}{l}-I M C \\
-C B \\
-D C T\end{array}$ & $\begin{array}{l}\text { O EN dos pacientes revelou predominância de } \\
\text { eutrofia no IMC. } \\
\text { Desnutrição na avaliação da DCT e da CB. }\end{array}$ \\
\hline
\end{tabular}

Legenda: EN: estado nutricional; CB: circunferência do braço; IMC: Índice de massa corporal; DCT: Dobra cutânea tricipital; ANSG: Avaliação Nutricional Subjetiva Global; NRS-2002: Escore de Risco Nutricional-2002; MUST: Instrumento Universal de Triagem de Desnutrição; TARV: terapia antirretroviral.

Fonte: Batista FKV, et al., 2020. 


\section{DISCUSSÃO}

Diante dos resultados nos estudos analisados a respeito do perfil nutricional de portadores de HIV/AIDS, pode-se constatar que, embora exista uma mudança no padrão corporal desse público, com o aumento de indivíduos eutróficos ou apresentando excesso de peso, permanece alto o número de pessoas que convivem com HIV/AIDS sendo diagnosticados com desnutrição. A desnutrição é caracterizada pela redução da composição corporal podendo acelerar a progressão da doença e consequentemente uma piora do estado funcional do portador do vírus, sendo esta considerada uma das maiores complicações no HIV+ (SOUZA CN, et al., 2017).

Tanto o baixo peso quanto o excesso de peso interferem de forma negativa, sendo capazes de agravar o quadro desses pacientes, pois um quadro de desnutrição favorece a fragilidade do sistema imune desse público, aumentando o risco que uma doença oportunista acometa esses indivíduos. Já o sobrepeso ou obesidade, facilita que essas pessoas desenvolvam alguma patologia crônica, por isso, a manutenção de um estado nutricional saudável é de extrema importância, pois possibilita amenizar tais riscos (SOUZA CN, et al., 2017).

No portador de HIV/AIDS a desnutrição pode ter causas diversas. Pessoas acometidas pelo vírus costumam ter uma ingestão alimentar reduzida, o que leva, consequentemente, a uma redução do peso do indivíduo. As causas para essa diminuição alimentar podem estar associadas a uma série de razões como: anorexia, fadiga, náuseas, vômitos, alterações metabólicas, diarreia, alterações neurológicas, fatores psicológicos, interação droga-nutriente, que gera deficiência de algumas vitaminas (A, C, B12, B6) e minerais como, zinco e selênio, assim, como o próprio tratamento medicamentos pode levar o paciente a esse quadro (PINTO AF, et al., 2016; SOUZA CN, et al., 2017; KAUFFMANN, et al., 2017).

O baixo peso interfere negativamente na qualidade de vida desses pacientes, visto que, indivíduos desnutridos relatam ter uma maior limitação em alguns domínios, incluindo limitação relacionada ao aspecto físico e a vitalidade, além disto, o baixo peso debilita o sistema imune, favorecendo o aparecimento de infecções oportunistas, o que nesse público deve ser evitado (OLIVEIRA RL, et al., 2018; RODRIGUES EC, et al., 2013).

Segundo Santana JC, et al. (2019) as doenças oportunistas são infecções que acometem o organismo humano devido alguma falha no sistema imunológico do indivíduo, sistema este que é responsável por liberar as células de defesa que combatem antígenos, ou seja, essas células tem a função de destruir corpos estranhos que invadem o corpo humano, ou seja, que não é pertencente aquele organismo, necessitando assim ser expulso do local em que estiver. Se o portador de HIV/AIDS estiver com o estado nutricional debilitado, essa condição irá favorecer a falha do sistema imunológico possibilitando, assim, o surgimento de infecções por não haver células capazes de destruir antígenos das diferentes doenças oportunistas, por estas estarem infectadas pelo vírus HIV e serem destruídas.

Monitorar o peso é imprescindível no acompanhamento nutricional do paciente portador de HIV/AIDS, pois esse monitoramento possibilita conhecimento de alterações nutricionais existentes, permitindo ao profissional um maior entendimento dessas complicações e por consequência, uma melhor adequação ao atendimento nutricional diante do vírus e das doenças oportunistas encontradas, visto que, como já citado a perda ponderal é um agravante que aumenta as chances de complicações relacionadas ao vírus (RODRIGUES EC, et al., 2013).

Nesses pacientes, a avaliação do estado nutricional tem como objetivo evitar ou reverter a desnutrição, oferecer um aporte adequado de nutrientes, reduzir os efeitos colaterais do tratamento medicamentoso, diminuir os sintomas de má-absorção, preservar a massa magra e promover qualidade de vida aos pacientes. Para se obter um diagnóstico fidedigno, é indispensável a avaliação antropométrica (circunferências e dobras cutâneas), visto que, desnutrição, imunodeficiência e infecção compõem uma tríade que deve ser evitada nesse público (SOUZA CN, et al., 2017).

Apesar dos resultados apresentados na maioria dos estudos ter prevalecido o estado nutricional de desnutrição, o diagnóstico nutricional de sobrepeso ou obesidade alto esteve presente em um número considerável de artigos, com grande parte da população analisada sendo diagnosticados com excesso de 
peso. As pesquisas atuais mostram que o uso da TARV, proporcionou uma redução de portadores de HIV/AIDS desnutridos, no entanto, ocorreu um aumentando de indivíduos apresentando excesso de peso. $O$ uso da TARV leva esses pacientes a apresentarem alteração no metabolismo dos lipídios e carboidratos, culminando na alteração na distribuição da gordura corporal, sendo esta, caracterizada por uma perda dos depósitos de gordura periférica (forma lipoatrófica) ou acúmulo de gordura central (forma lipohipertrófica) ou ambas as alterações (forma mista), que a médio e longo prazo comprometem a saúde física e psíquica, bem como a adesão ao tratamento (AIRES IO, et al., 2019; DUTRA CDT, et al., 2011).

Atualmente, o ganho de peso, que por muitas vezes leva a um quadro de obesidade e a redistribuição de gordura, são novos dilemas nutricionais que os indivíduos com HIV/AIDS em uso de terapia TARV estão manifestando. Mesmo que o ganho de peso seja benéfico ao sistema imune, no entanto, atrelado a esse aumento ponderal, houve um crescimento no aparecimento de patologias crônicas não transmissíveis e comorbidades associadas ao uso da terapia antirretroviral. O excesso de gordura em pessoas que vivem com HIV/AIDS configura-se como um fator adicional para resistência insulínica e o desenvolvimento de diabetes mellitus (AIRES IO, et al., 2019).

Além disso, esse aumento ponderal tem sido associado ao aumento da pressão arterial nessa população. Os mecanismos que relacionam a obesidade com a hipertensão são complexos e o tratamento dessa patologia em indivíduos obesos é um desafio para os pacientes e profissionais da área da saúde. Estudos relatam que o maior risco para hipertensão em indivíduos que convivem com o HIV está associado a Lipodistrofia. $O$ excesso de peso se for atrelado a hábitos inadequados pode ocasionar desordens metabólicas complicando o estado de saúde dos pacientes, ademais, o uso de TARV associado ao sobrepeso ou obesidade e ao acúmulo de gordura é um preditor para que o paciente possa vir a desenvolver síndrome metabólica (ARRUDA CM e PEREIRA PCM, 2015; SILVA VS et al., 2012).

A avaliação do estado nutricional no paciente com HIV/AIDS é de grande importância, pois existem relações entre estado nutricional e evolução da doença. Anteriormente ao uso da TARV, os indivíduos com HIV/AIDS eram comumente acometidos por desnutrição e carências nutricionais. Hoje, observa-se uma mudança nesse quadro, o uso da TARV tem contribuído para uma modificação do perfil nutricional desses indivíduos. Nessa revisão os resultados dos estudos mostraram que houveram elevados números de indivíduos com eutrofia e excesso de peso em uso de TARV. Esse aumento de casos de eutrofia, sobrepeso ou obesidade em pacientes portadores de HIVIAIDS em uso de TARV pode se justificar pela capacidade que alguns antirretrovirais, como os inibidores de protease, têm de alterar o perfil lipídico e contribuir para uma mudança na redistribuição de gordura corporal (LADEIRA POC e SILVA DCG, 2012; COSTA CS, et al., 2017).

Os resultados encontrados na literatura são importantes e precisam ser levados em consideração no momento da intervenção dos vários profissionais de saúde envolvidos no tratamento e acompanhamento desses pacientes, dentre eles o nutricionista. Neste sentido, a intervenção nutricional torna-se uma importante ferramenta no tratamento desses pacientes, uma vez que hábitos alimentares saudáveis são fundamentais no controle do peso e na manutenção dos parâmetros bioquímicos em níveis adequados (SILVA VS, et al., 2012).

A avaliação nutricional ajuda identificar possíveis riscos nutricionais, a melhorar o sistema imunológico e a controlar alterações gastrointestinais e patologias associadas que debilitam e se aproveitam, muitas vezes, do estado imunológico fragilizado do indivíduo. É fundamental, portanto, a realização de novas pesquisas abordando o tema, bem como a implementação de ações de educação alimentar e nutricional, sabendo que, a educação alimentar e nutricional tem como função auxiliar as pessoas quanto à adoção de hábitos alimentares benéficos, educando esses indivíduos para que os mesmos possam ser capazes de realizarem escolhas alimentares adequadas e que proporcionem refeições que contemplem a base de uma alimentação saudável (LADEIRA POC e SILVA DCG, 2012).

A intervenção nutricional tem a capacidade de melhorar a resposta ao tratamento e, assim, reduzir o quadro de desnutrição ou de excesso de peso, proporcionado uma melhora na qualidade de vida desses pacientes. Nos estudos em que os autores utilizaram mais de um método para realizar o diagnóstico 
nutricional, foi possível perceber que em alguns artigos, os métodos utilizados discordaram no diagnostico, essa informação reforça a importância de utilizar mais de uma medida no momento da avaliação, a fim de que se tenha um diagnóstico mais preciso do estado nutricional do avaliado (DUTRA CDT, et al., 2011).

Essa discordância se deu principalmente entre as medidas IMC, CB e DCT, onde IMC na maioria dos estudos classificou que os indivíduos estavam eutroficos, enquanto, CB e DCT diagnosticaram algum grau de desnutrição. A disparidade encontrada nos resultados, podem estar relacionadas a alterações na forma corporal, onde ocorre hipertrofia adiposa central e lipotrofia periférica, conhecida como lipodistrofia associada TARV. A circunferência é composta pela soma dos tecidos ósseo, muscular, gorduroso e epitelial. Logo, a diminuição dessa circunferência reflete a perda do conteúdo protéico, bem como de tecido adiposo, além de ser uma medida sensível à depleção de massa muscular (PINTO AF, et al., 2016; SILVA AAA, et al., 2015).

A principal limitação dessa revisão consiste na escassez de estudos dessa natureza com o objetivo de investigar o perfil nutricional desse público. Frente aos resultados desta revisão, observa-se a necessidade de estratégias a nível de saúde pública que busquem realizar avaliação nutricional nos portadores de HIV/AIDS, visto que, ainda é grande o número de pacientes que estão em risco nutricional, sendo importante manter ou recuperar o estado nutricional desse público.

\section{CONSIDERAÇÕES FINAIS}

Embora exista uma mudança no padrão corporal dessa população, com o aumento de indivíduos com sobrepeso ou obesidade, a desnutrição permanece prevalente, sendo necessário uma atenção das políticas públicas de saúde, dado que, tanto o peso elevado quanto o baixo peso são bastantes prejudicais, principalmente nessa população, pois, a desnutrição debilita o sistema imune, o que deixa o paciente suscetível a infecções oportunistas e o excesso de peso possibilita o surgimento de outras patologias. Desse modo, o monitoramento do estado nutricional torna-se indispensável nesse público, para que se possa detectar distúrbios nutricionais precocemente, e assim, realizar uma intervenção adequada.

\section{REFERÊNCIAS}

1. AIRES IO, et al. Aspectos clínicos e nutricionais em pessoas vivendo com HIV/AIDS: uma série de casos. Revista Eletrônica Acervo Saúde, 2019; sup.28: e1077.

2. ARRUDA CM, PEREIRA PCM. Pacientes com HIV/AIDS em uso de inibidores de protease e a relação entre estado nutricional e hipertensão arterial sistêmica. Revista de Ciências Médicas, 2015; 24(1): 11-18.

3. BASSICHETTO KC, et al. Fatores associados à desnutrição em pessoas com 20 anos e mais, com HIV/AIDS, em serviços públicos de saúde no Município de São Paulo, Brasil. Caderno de Saúde Pública, 2014; 30(12): 1-9.

4. BRAGA LA, SILVA CAB. Avaliação nutricional e metabólica de pacientes com HIV em uso da terapia antirretroviral no Nordeste do Brasil. Revista Brasileira em Promoção da Saúde, 2010; 23(4): 368-373.

5. BRASIL, MS. Boletim Epidemiológico de HIV e AIDS. Secretaria de Vigilância em Saúde, 2019.

6. COSTA CF, et al. Associação entre diferentes métodos de avaliação nutricional em pacientes com HIV/AIDS em um hospital público. Revista Brasileira em Promoção da Saúde, 2017; 30(3): 1-9.

7. COSTA CS, et al. Associação entre diferentes métodos de avaliação nutricional em pacientes com HIV/AIDS em um hospital público. Revista Brasileira em Promoção da Saúde, 2017; 30(3): 1-9.

8. DUTRA CBT, et al. Avaliação do consumo alimentar em pacientes HIV positivos com Lipodistrofia. Revista Ciência \& Saúde, 2011, 4(2): 59-65.

9. KAMIMURA MA, et al. Avaliação nutricional. In: Cuppari L. Guias de medicina ambulatorial e hospitalar: nutrição clínica do adulto. 3 ed. Barueri, SP: Manole, 2014; 71-109p.

10. KAUFFMANN LKO, et al. Perfil nutricional e alimentar de portadores de HIV-1/AIDS internados em um hospital universitário. Ciência \& Saúde, 2017; 10(2): 82-88.

11. KROLL AF, et al. Prevalence of obesity and cardiovascular risk in patients with HIV/AIDS in Porto Alegre, Brazil. Arquivos Brasileiros de Endocrinologia \& Metabologia, 2012; 56(2): 137-141.

12. LADEIRA POC, SILVA DCG. Estado Nutricional e Perfil Alimentar de Pacientes Assistidos pelo Programa de DST/AIDS e Hepatites Virais de um Centro de Saúde de Itaperuna-RJ. Jornal Brasileiro de Doenças Sexualmente Transmissíveis, 2012; 24(1):28-31.

13. MIRANDA RNA, et al. Caracterização nutricional de pacientes com HIV/AIDS coinfectados ou não com tuberculose internados no hospital universitário em Belém, estado do Pará. Revista Eletrônica Acervo Saúde, 2019; Sup.18: e976.

14. OLIVEIRA N, et al. Anthropometric measures as indicators of the nutritional status of people living with HIV. Revista chilena de nutrición, 2019; 46(6): 753-760. 
15. OLIVEIRA RL, et al. Desnutrição e Qualidade de Vida em Pessoas Vivendo com HIV/AIDS. Revista Brasileira de Ciências da Saúde, 2018, 22(1): 65-72.

16. PEREIRA MD, et al. Esquema terapêutico e consumo alimentar em pessoas vivendo com HIV/AIDS.Archives Of Health Investigation, 2019; 8(7): 349-356.

17. PINTO AF, et al. Estado nutricional e alterações gastrointestinais de pacientes hospitalizados com HIV/AIDS no Hospital Universitário João de Barros Barreto em Belém, Estado do Pará, Brasil. Revista Pan-Amazônica de Saúde, 2016; 7(4): 47-52.

18. PIRES DS, et al. Perfil nutricional e métodos de avaliação do estado nutricional de pacientes infectados pelo HIV. BRASPEN, 2017; 32(3): 209-213.

19. RODRIGUES EC, et al. Avaliação do perfil nutricional e alimentar de portadores do HIV. Revista Paraense de Medicina, 2013; 27(4): 37-46.

20. SANTANA JC, et al. Principais doenças oportunistas em indivíduos com a HIV. Revista Multidisciplinar, 2019; 16(13): 405-422.

21. SILVA AAA, et al. Prevalência de má nutrição e doenças oportunistas em pacientes hiv/AIDS internados em um hospital referência em Porto Velho - Rondônia. Revista Saber Científico, 2015; 4(1): 58-64.

22. SILVA VS, et al. Alterações Nutricionais em Pacientes com Lipodistrofia Associada ao HIV/AIDS de uma Unidade de Referência do Município de Belém - Pará. Jornal Brasileiro de Doenças Sexualmente Transmissíveis, 2012; 24(4): 233-238.

23. SOUZA CN, et al. Perfil nutricional de pacientes HIV/AIDS hospitalizados. Revista Multitemas, 2018; 23(53):159-181. 